\title{
Der Publikumstag
}

Erhard Taverna

\footnotetext{
* Publikumstag «EXIT 30 Jahre für die Selbstbestimmung» am 15. Juni 2012 in Oerlikon.
}

Die angekündeten Gegendemonstrationen sind ausgeblieben. Nur wenige Menschen haben die Konferenz der Euthanasia Prevention Coalition (EPC) vis-à-vis besucht. Vielleicht war es das schöne Sommerwetter. Im regen Verkehr vor dem Bahnhof Oerlikon verteilen zwei junge Leute eine Broschüre der Human Life International, wer will, darf ein Medaillon der «Legion Mariens» nach Hause tragen. Zwei Sicherheitsleute stehen gelangweilt am Eingang zum Swissôtel, denn der gutbesuchte Publikumstag der World Federation of Right to Die Societies (WFRtDS) verläuft ungestört. Exit Schweiz feiert sein 30-JahreJubiläum mit Referenten aus der Schweiz, Deutschland, Grossbritannien, Kanada, USA, Belgien, Frankreich und Australien*. Ein konzentrierter Vortragsparcours auf beachtlichem Niveau, angereichert durch Podiumsgespräche, moderiert von Erich Gisling, Journalist und Buchautor, und zusammenfassende Kommentare von Karl Lüönd, Korrespondent und Sachbuchautor. Ergänzend bietet ein Büchertisch eine breite Auswahl, darunter auch das Büchlein «Ausgang» von Ueli Oswald, Sohn des bekannten Unternehmers und Militärs. Er spricht aus seiner Erfahrung als Referent zu den Anliegen der Angehörigen.

\section{Wechselvolle Geschichte}

Die wechselvolle Geschichte von EXIT Schweiz erreichte im Mai 2011 einen Höhepunkt, als im Kanton Zürich $84,5 \%$ der Stimmberechtigten eine Volksinitiative der EDU ablehnten, die jede Art von Sterbehilfe unter Strafe stellen wollte. Die Zahl der Vereinsmitglieder liegt heute bei über 60000 in der deutschen Schweiz und über 20000 in der Romandie. Die erste Suizidbegleitung fand 1985 statt, 2001 waren es 178, im Jahr 2011465 Begleitungen, Tendenz weiterhin steigend (zum Vergleich: In Deutschland wurden 2011 offiziell 27 begleitete Suizide registriert). Pflegt die Schweiz mit Exit und dem «Suizidtourismus» von Dignitas eine Todeskultur, wie die vorwiegend katholischen Gegner behaupten? 1983 wollte Exit ein Sterbehospiz, ausdrücklich ohne Freitodhilfe, gründen, ein Projekt, das nach mehreren Anläufen an Einsprachen und fehlenden öffentlichen Subventionen scheiterte. Aus heutiger Sicht eine Pioniertat auf dem Weg zum Aufbau der Palliativmedizin, die seither auch durch die Exit-Stiftung «palliacura» aktiv gefördert wird. Der leitende Oberstaatsanwalt des Kantons Zürich, Andreas Brunner, unterscheidet klar zwischen Sterbehilfe, als Hilfe beim Sterben, und Suizidhilfe, als Hilfe zum Sterben. Sein klares Ja zur organisierten Suizidhilfe, verbunden mit einem Ja zu Qualitätssicherung und Rechtssicherheit, führte zu

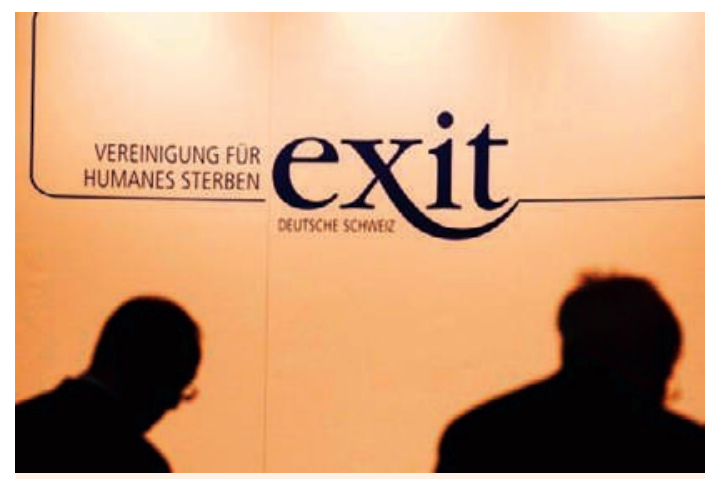

Sein 30-Jahre-Jubliäum feierte Exit Schweiz mit einem «Publikumstag».

einer kantonalen Vereinbarung mit gemeinsam erarbeiteten Rahmenbedingungen, die 2010, aufgrund einer Beschwerdekoalition, durch das Bundesgericht abgelehnt wurde. Die gleichen Kreise versuchten, über die Bundesrätin Eveline Widmer-Schlumpf eine Kehrtwende zu erzielen. Wobei sowohl eine einengende Variante wie ein vollständiges Verbot in der folgenden Vernehmlassung keine Chance hatten.

\section{Interessante Referate, spannende Diskussionen}

Die am Nachmittag anwesende Bundesrätin Simonetta Sommaruga distanziert sich von ihrer Vorgängerin, indem sie unmissverständlich die freie Selbstbestimmung befürwortet: sowohl weitere Palliativangebote als auch organisierte Suizidhilfe. Der Staatsanwalt stellt die aktuelle Leidensfähigkeit oder Leidensbereitschaft in Frage, was im Plenum eine interessante Diskussion auslöst. Sind wir verweichlicht? Die Frage provoziert Antworten. Es wird moniert, dass Autonomieverlust, passives Geschehenlassen und Ausgeliefertsein uns schwerfalle, und dass Erdulden ohne religiöse Gewissheit an Sinn verloren habe. Niemand wünscht sich das harte Leben früherer Generationen zurück. Bemerkt wird aber auch, dass mit den Möglichkeiten der Medizin, mit der Lebensdauer auch die Dauer des Leidens zugenommen hat. Die Medizin rettet Leben auch dann, wenn die Folgen unabsehbar sind. Sie erzeugt, wenn auch ungewollt, in vielen Fällen lebenslängliche Behinderungen und chronische Krankheiten. Mit Fallbeispielen und einer kleinen Statistik verdeutlicht der Palliativmediziner Andreas Weber, worum es geht. Jeder Fall liegt anders, wichtig ist die Information der Betroffenen und der Angehörigen. Es macht keinen Sinn, die unterschiedlichen Angebote gegeneinander auszuspielen. Kritik gibt es trotzdem: Letztlich 
mache es keinen grossen Unterschied, ob jemand Natriumpentobarbital zu sich nehme oder unter einer Dauersedation mit Dormicum seinen Tod finde. Das eine hat einen klaren Termin, das andere bedeutet einen mehr oder weniger spontanen $\mathrm{Ab}$ schluss vieler Vorstufen.

Die Palliativmedizin hat es leichter. Sie darf teuer sein und den Berufstätigen ein Einkommen sichern. Dass es im Unterschied dazu keinen gesetzlichen Anspruch auf Suizidhilfe gibt, macht die freiwilligen und ehrenamtlichen Helfer, trotz verhöramtlicher Kontrolle, immer etwas verdächtig. Gutdokumentiert ist an der Tagung auch die Organisation Dignitas, die sich 1998 von Exit abspaltete. Der Begründer, Rechtsanwalt Ludwig A. Minelli, argumentiert überzeugend für eine «Suizidversuchs-Prophylaxe», die auch unvoreingenommen den Suizid als Möglichkeit mitdenkt. Er kritisiert die Wissenschaft und die Medien, die sich bisher für die weit bedeutsamere Frage nach der suizidpräventiven Wirkung der in diesem Feld tätigen Organisationen wenig interessiert habe.
Spannend sind die Referate zur Situation in anderen Ländern und zum Europarat, in dem kirchliche Kreise intensiv lobbyieren. Der anglikanische Pastor James Woodword, Mitglied der FalconerCommission, spricht sich in England gegen den assistierten Suizid aus, weil er befürchtet, dass der Staat diesen für einen noch weiteren Abbau von Leistungen des Gesundheitswesens missbrauchen würde. Dr. med. Philip Nitschke, Arzt und Buchautor, zieht aus der gegenwärtig repressiven Gesetzgebung in Australien andere Schlüsse. Mit seinem im Lande verbotenen Buch «The Peacefull Pill», einer detaillierten Do-it-yourself-Suizid-Anleitung, betont er den niederschwelligen Zugang für jedermann, bei möglichst minimaler Gesetzgebung. In einer globalisierten Wirtschaft sei auch Nembutal immer erhältlich. «The winding road to a good death», so der Titel des letzten Podiums. Es gibt keine einfachen Antworten. Für einmal hat die Schweiz eine vorbildliche Rolle eingenommen. Wo es eine direkte Demokratie gibt, entscheiden mündige Bürgerinnen und Bürger. Dafür werden wir vielerorts zu Recht beneidet. 